\title{
基于亲电氯代反应的次氯酸荧光探针构建及细胞成像研究
}

\author{
赵 云* 李艳芳李蓉晓王雅卿樊晓霞 \\ (太原师范学院 山西晋中 030619)
}

\begin{abstract}
摘要 设计并合成了一种简单的由吩噻嗪-蒱酮共轭的新型次氯酸荧光探针 PIOCI. 该探针实现了在生理条件下，基于 氯正离子 $\left(\mathrm{Cl}^{+}\right)$引发独特亲电氯代反应机制的 $\mathrm{HOCl}$ 检测. 实验结果表明, 探针 PIOCI 对 HOCl 具有荧光 “关-开” 型响 应, 检测前后苂光强度增加了 600 多倍, $\mathrm{HOCl}$ 浓度在 $0 \sim 6.0 \mu \mathrm{mol} / \mathrm{L}$ 呈良好的线性关系 $\left(R^{2}=0.997\right)$, 检测限达 1.40 $\mathrm{nmol} / \mathrm{L}$. 可实现低至 $0.25 \mu \mathrm{mol} / \mathrm{L}$ 的裸眼检测以及快速响应 $(<3 \mathrm{~s})$. 通过质谱实验、光谱实验和理论计算验证了 $\mathbf{P I O C I}$ 对 $\mathrm{HOCl}$ 的识别机理. 利用苂光成像技术, 成功对细胞内源性和外源性 HOCl 进行了检测, 表明探针 PIOCl 在生物学上 具有潜在的应用前景.
\end{abstract}

关键词 次氯酸; 苂光探针; 亲电氯代; 茚酮; 荧光增强

\section{A New Fluorescent Probe for Hypochlorous Acid Based on Chlorinium lons Recognition Mechanism and Its Bioimaging Research in Living Cells}

\author{
Zhao, Yun* Li, Yanfang Li, Rongxiao Wang, Yaqing Fan, Xiaoxia \\ (Department of Chemistry, Taiyuan Normal University, Jinzhong, Shanxi 030619)
}

\begin{abstract}
A simple phenothiazine-indanone conjugated fluorescent probe PIOCl for $\mathrm{HOCl}$ was designed and synthesized. For the first time, a new fluorescent probe for $\mathrm{HOCl}$ based on $\mathrm{Cl}^{+}$induced the unique electrophilic substitution reaction under the physiological conditions. The probe PIOCl was a turn-on response to $\mathrm{HOCl}$, and the fluorescence intensity increased more than 600 times after addition of $\mathrm{HOCl}$. In the range of $\mathrm{HOCl}$ concentration from 0 to $6.0 \mu \mathrm{mol} / \mathrm{L}$, the fluorescence intensity had a good linear relationship with $\mathrm{HOCl}$ concentration $\left(R^{2}=0.997\right)$, the detection limit was $1.40 \mathrm{nmol} / \mathrm{L}$, and the naked eye detection as low as $0.25 \mu \mathrm{mol} / \mathrm{L}$ and rapid response $(<3 \mathrm{~s})$ can be achieved under $1.0 \mu \mathrm{mol} / \mathrm{L} \mathrm{HOCl}$. The sensing mechanism of probe PIOCI for $\mathrm{HOCl}$ was verified by NMR, HRMS and density function theory calculations. Using fluorescence imaging technology, the endogenous and exogenous $\mathrm{HOCl}$ has been successfully detected, which indicates that probe PIOCI has potential application prospects in analytical detection and pathological analysis.

Keywords hypochlorous acid; fluorescent probe; electrophilic chlorination; indanone; turn-on mechanism
\end{abstract}

次氯酸 $(\mathrm{HOCl})$ 作为内源性活性氧物质之一，在生物 体内可由过氧化氢和氯离子在髓过氧化物酶(MPO)催 化作用下产生 ${ }^{[1]}$. 研究发现, HOCl 介导多种生理过程, 在机体抵抗病原免疫防御、调节细胞内氧化还原平衡、 激活转录因子等过程中起着至关重要的作用 ${ }^{[2-3]}$. 同时, $\mathrm{HOCl}$ 具有强氧化性，能够与多种生物分子发生反应, 比如 DNA、RNA、脂类、蛋白质、铁硫族合物和硫醇 等 ${ }^{[4]}$. 近年来, 越来越多的研究表明, 细胞内过度表达
的 $\mathrm{HOCl}$ 会导致细胞和组织器官的调亡和损伤，进而引 发多种疾病, 如动脉弹样硬化、肝损伤、类风湿性关节 炎、癌症等 ${ }^{[5-7]}$. 因此, 快速和灵敏地检测生物环境中的 $\mathrm{HClO}$ 在疾病诊断和预防方面具有重要意义.

相比于传统的检测方法，由荧光团和识别基团构筑 的苂光探针法，因具有灵敏度高、特异性强、操作简单、 响应时间快以及实时检测等优点, 在多种生物活性分子 和离子的化学传感、活体动态监测等方面得到了广泛应

\footnotetext{
* Corresponding author. E-mail: zhaoyun19830317@163.com

Received January 22, 2021; revised March 15, 2021; published online April 2, 2021

Project supported by the National Natural Science Foundation of China (No.21807077), the Shanxi Provincial Natural Science Foundation for Youths (No. 201801D221081), the Scientific and Technological Innovation Programs of Higher Education Institutions in Shanxi Province (No. 201802100).

国家自然科学基金(No. 21807077)、山西省自然科学青年基金(No. 201801D221081)、山西省高等学校科技创新(No. 201802100)资助项目
} 
用 ${ }^{[8]}$. 近些年, 化学工作者已成功开发了一些应用于 $\mathrm{HOCl}$ 检测的良好有机小分子苂光探针 ${ }^{[9-11]}$. 这些探针 大多利用 $\mathrm{HOCl}$ 的氧化性作为反应原理而设计. 目前报 道的主要有氧化脱肜机理、氧化含硫族元素 $(\mathrm{S} 、 \mathrm{Se} 、 \mathrm{Te}$ 元素)原子或基团机理、氧化对甲基苯酚或对甲氧基苯 胺机理、氧化胺成硝基、脱硫环化、氧化碳碳双键、氧 化脱亚氨基马来腈等类型 ${ }^{[12-13]}$. 虽然, 利用 $\mathrm{HOCl}$ 的强 氧化性设计的反应型探针可以实现 $\mathrm{HOCl}$ 的选择性识 别. 但是有时也易受到其它具有更强氧化性的生物客体 的干扰, 如超氧阴离子 $\left(\mathrm{O}_{2}^{-\cdot}\right)$ 、过氧亚硝基 $\left(\mathrm{ONOO}^{-}\right)$ 等 ${ }^{[14-20]}$. 因此, 寻找在生理条件下对 $\mathrm{HOCl}$ 具有更高选 择性、更高灵敏度和更短响应时间的特异性检测方法仍 然是一项有吸引力的工作.

本工作组设计并合成了一种简单的吩噻嗪-茚酮共 轭苂光探针 PIOCI, 如 Scheme 1 所示. 通过在狮酮分子 中引入大 $\pi$ 共轭的供电子基团, 提高探针与 $\mathrm{HOCl}$ 反应 活性, 实现了在生理条件下, 高选择性和高灵敏性的 $\mathrm{HOCl}$ 检测. 检测前后荧光强度增加了 600 多倍, 检测限 达到 $1.40 \mathrm{nmol} / \mathrm{L}$. 同时还可实现低至 $0.25 \mu \mathrm{mol} / \mathrm{L}$ 的裸 眼检测以及快速响应 $(<3 \mathrm{~s})$. 质谱实验、光谱实验和密 度泛函理论计算验证了探针 PIOCI 对 HOCl 的识别机理 为亲电氯代反应机制, 如 Scheme 1 所示. 在 HeLa 细胞 中进行的 $\mathrm{HOCl}$ 苂光成像实验, 实现了内源性和外源性 HOCl 的苂光检测, 表明探针 PIOCI 在生物学上具有良 好的应用前景应用.

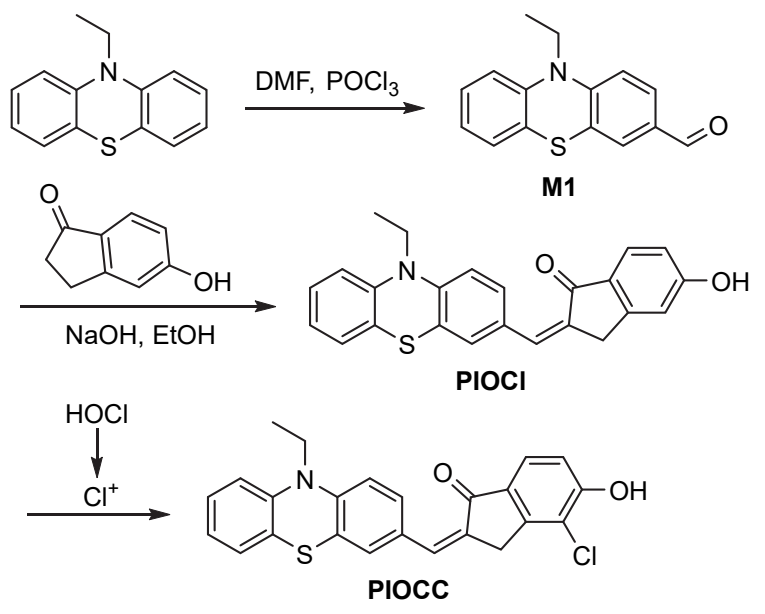

图式 1 苂光探针 PIOCI 的合成路线及机理 Scheme 1 Synthesis and Proposed mechanisms of probe PIOCI

\section{1 结果与讨论}

\section{1 探针 $\mathrm{PIOCI}$ 的设计与合成}

与其它生物活性氧和氮相比, $\mathrm{HOCl}$ 作为一种弱酸 $\left(\mathrm{p} K_{\mathrm{a}}=7.54\right)$, 在生理条件下 $\mathrm{HOCl}$ 分子可以分解出氯正 离子 $\left(\mathrm{Cl}^{+}\right) \cdot \mathrm{Cl}^{+}$具有强的亲电性, 能够与芳香体系发生特
异性亲电氯代反应 ${ }^{[21]}$. 因此, 将 $\mathrm{HOCl}$ 与本身具有大 $\pi$ 共轭芳香体系的有机小分子探针发生亲电氯代反应，可 改变苂光探针的电子云分布, 实现对 $\mathrm{HOCl}$ 的高选择性 检测. 基于上述设计策略, 我们以 5-着基-1-狮酮为识别 基团, 吩噻嗪为供电子基团, 构建出新型 $\mathrm{HOCl}$ 荧光探 针 (Z)-2-( $N$-乙基-3-亚甲基吩噻嗪基)-5-羟基-1-狮酮 (PIOCI, Scheme 1). 探针经过两步简易的化学反应合成 制得：以 10-乙基吩噻嗪为原料，通过 Vilsmeier-Haack 反应得到中间体 M1, 然后, 在乙醇溶液中将中间体 M1、 $\mathrm{NaOH}$ 与 5-差基-1-狮酮加热回流得到探针 PIOCI. 利用高分辨质谱(HRMS)、核磁共振氢谱 $\left({ }^{1} \mathrm{H}\right.$ NMR)、核 磁共振碳谱 $\left({ }^{13} \mathrm{C} \mathrm{NMR}\right)$ 对所合成的化合物进行了结构表 征.

\section{2 探针 PIOCI 对 $\mathrm{HOCl}$ 的光谱性质研究}

获得探针分子后, 首先对探针 PIOCl 与 $\mathrm{HOCl}$ 响应 的检测体系进行了篮选. 将 PIOCl $(10 \mu \mathrm{mol} / \mathrm{L})$ 与 $\mathrm{HOCl}$ 分别在水溶性溶剂甲醇 $(\mathrm{MeOH}) 、 乙$ 醇 $(\mathrm{EtOH}) 、 N, N$-二 甲基甲酰胺(DMF)、二甲基亚砜( DMSO)、乙腈 $\left(\mathrm{CH}_{3} \mathrm{CN}\right)$ 与磷酸盐缓冲溶液 (PBS, pH 7.4, $20 \mathrm{mmol} / \mathrm{L}$ ) 等体积混合 液中进行苂光光谱测试, 结果显示探针 PIOCI 在质子性 溶剂甲醇和乙醇混合液中表现出更高的苂光发射峰. 随 后, 将探针 PIOCI 在乙醇与不同比例的磷酸盐缓冲溶液 (PBS, pH 7.4, $20 \mathrm{mmol} / \mathrm{L}$ ) 体系进行荧光光谱测试, 发现 当乙醇含量超过 $80 \%$ 时, 苂光发射峰出现了明显的下 降. 以上结果表明探针 PIOCI 显示出与 5-羟基-1-狮酮 类染料共有的反溶剂效应 ${ }^{[22]}$. 因此选择在 PBS (20 $\mathrm{mmol} / \mathrm{L}, \mathrm{pH}=7.4,30 \%$ EtOH) 中进行探针 PIOCI $(10$ $\mu \mathrm{mol} / \mathrm{L})$ 与 $\mathrm{HOCl}$ 作用的光谱研究. 如图 $1 \mathrm{a}$ 所示, 反应前 探针 PIOCI 的最大吸收峰在 $435 \mathrm{~nm}$ 附近; 加入 $\mathrm{HOCl}$ 后, 溶液在 $410 \mathrm{~nm}$ 处出现一个新的吸收峰, 且随着 $\mathrm{HOCl}$ 浓度的增加而增强, 同时在 340 和 $435 \mathrm{~nm}$ 分别形 成等吸收点, 显示有新产物形成. 最大吸收峰的蓝移, 表明 $\mathrm{HOCl}$ 的氯代反应降低了茚酮酚羟基的供电子能 力, 使得氢键更易形成, 从而最大限度地减少激发态能 量消耗. 随后, 以 $420 \mathrm{~nm}$ 为激发波长, 检测了探针 PIOCl 与 $\mathrm{HOCl}$ 反应前后的荧光光谱变化. 未加入 $\mathrm{HOCl}$ 时, 探针 PIOCI 在 $514 \mathrm{~nm}$ 处几乎无荧光; 随着 $\mathrm{HOCl}$ 浓度不断增加 $(0 \sim 10 \mu \mathrm{mol} / \mathrm{L}), 514 \mathrm{~nm}$ 处的苂光强度逐 渐增强(图 1b), 并且在 1.0 倍量 $\mathrm{HOCl}$ 时苂光增强达到稳 定, 荧光强度增加 600 倍. 苂光实验表明探针 PIOCI 是 一个灵敏的苂光增强型探针. 通过选取 $514 \mathrm{~nm}$ 处的苂 光强度与 $\mathrm{HOCl}$ 浓度的变化作图可以看出, 测试体系的 荧光强度与 $\mathrm{HOCl}$ 浓度在 $0 \sim 6 \mu \mathrm{mol} / \mathrm{L}$ 范围内具有较好 的线性关系 $\left(R^{2}=0.9971\right)$. 经计算得出检测限低至 $1.40 \mathrm{nmol} / \mathrm{L}\left(3 \sigma / \kappa^{\text {法 }}\right)^{[10]}$. 另外, 探针对低浓度的 $\mathrm{HOCl}$ 也 

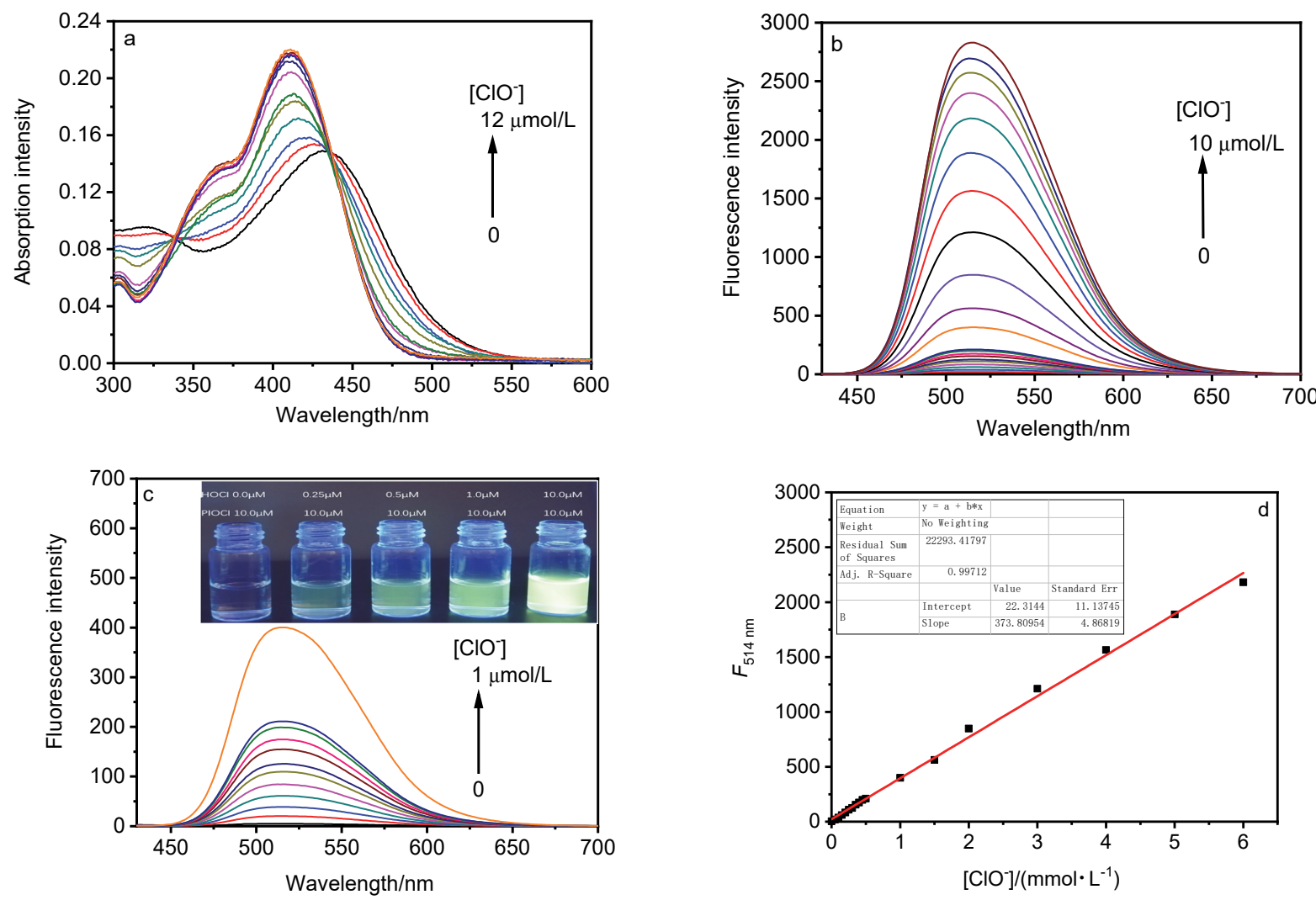

图 1 在 $\mathrm{PBS}$ 缓冲溶液 $(20 \mathrm{mmol} / \mathrm{L}, \mathrm{pH}=7.4,30 \% \mathrm{EtOH})$ 中探针 PIOCl $(10 \mu \mathrm{mol} / \mathrm{L})$ 与不同浓度的 $\mathrm{HOCl}(0 \sim 10 \mu \mathrm{mol} / \mathrm{L})$ 的(a)紫外可见光吸收光谱和(b)荧光发射光谱, (c)探针 PIOCI $(10 \mu \mathrm{mol} / \mathrm{L})$ 与低浓度 $\mathrm{HOCl}(0 \sim 1.0 \mu \mathrm{mol} / \mathrm{L})$ 的苂光发射光谱[插图: 探针 PIOCl $(10 \mu \mathrm{mol} / \mathrm{L})$ 分别与 $0.0 、 0.25 、 0.5 、 1.0$ 和 $10.0 \mu \mathrm{mol} / \mathrm{L} \mathrm{HOCl})$ 在 $365 \mathrm{~nm}$ 紫外灯照射下的照片], 以及(d)探针 PIOCI 在 $514 \mathrm{~nm}$ 处荧光强度与 $\mathrm{HOCl}$ 浓度的线性相关性 $\left(\lambda_{\mathrm{ex}}=420 \mathrm{~nm}\right)$

Figure 1 In PBS buffer ( $\mathrm{pH}=7.4,10 \mathrm{mmol} / \mathrm{L}, 30 \% \mathrm{EtOH})$, absorption spectra (a) and fluorescence spectra (b) of probe PIOCl (10 $\mu \mathrm{mol} / \mathrm{L})$ in response to $\mathrm{HOCl}(0 \sim 10 \mu \mathrm{mol} / \mathrm{L})$, (c) fluorescence spectra at low concentration of $\mathrm{HOCl}(0 \sim 1.0 \mu \mathrm{mol} / \mathrm{L})$ (insert: the color changes after adding $\mathrm{HOCl}$ under $365 \mathrm{~nm}$ UV-light), and (d) the linear relationship between the fluorescence intensity at $514 \mathrm{~nm}$ and the concentration of $\mathrm{HOCl}(0 \sim 6 \mu \mathrm{mol} / \mathrm{L})$

具有非常好的线性识别和较高的灵敏度, 能够对 0.25 $\mu \mathrm{mol} / \mathrm{L}$ 的 $\mathrm{HOCl}$ 实现裸眼观察, 如图 1c 所示. 这些数据 表明，探针 PIOCI 对 HOCl 具有较高的信噪比和灵敏度, 适用于样品中微量 $\mathrm{HOCl}$ 的检测.

\section{3 苂光动力学响应及探针光稳定性能研究}

探针对客体的响应速度是评价荧光探针法性能的 一个关键因素. 因此, 对探针 PIOCI 与 $\mathrm{HOCl}$ 相互作用 的时间依赖性进行了研究. 如图 2a 所示, 探针 PIOCI $(10 \mu \mathrm{mol} / \mathrm{L})$ 和不同浓度的 $\mathrm{HOCl}(0,1.0,2.0,5.0,10.0$ $\mu \mathrm{mol} / \mathrm{L})$ 在 PBS $(20 \mathrm{mmol} / \mathrm{L}, \mathrm{pH}=7.4,30 \% \mathrm{EtOH})$ 中相互 作用. 结果表明, 即使 $\mathrm{HOCl}$ 浓度低至 $1.0 \mu \mathrm{mol} / \mathrm{L}$, 探针 PIOCI 也具有快速的响应 $(<3 \mathrm{~s})$, 并且继续延长反应时 间, 反应体系的荧光强度波动也不大, 表明探针 $\mathbf{P I O C I}$ 对 $\mathrm{HOCl}$ 具有良好的识别速度和光稳定性.

\section{4 探针 $\mathrm{PIOCI}$ 在不同 $\mathrm{pH}$ 溶液中对 $\mathrm{HOCl}$ 的识别研} 究

为了研究 $\mathrm{pH}$ 对探针 PIOCl 与 $\mathrm{HOCl}$ 荧光响应的影
响, 在 $\mathrm{pH} 4.0 \sim 10.0$ 范围内分别对探针 PIOCI、探针 PIOCI 与 $\mathrm{HOCl}$ 作用后的荧光强度进行检测. 如图 $2 b$ 所 示, 未添加 $\mathrm{HOCl}$ 时, 溶液几乎没有荧光响应, 表明探 针在生理 $\mathrm{pH}$ 环境下比较稳定. 当加入 $\mathrm{HOCl}(10$ $\mu \mathrm{mol} / \mathrm{L}$ )反应后, 在 $\mathrm{pH} 6 \sim 8$ 范围内表现出非常显著的荧 光响应, 并且在 $\mathrm{pH}=7$ 时达到最大值. 因此, 探针 PIOCI 可以用于生理条件下的 $\mathrm{HOCl}$ 检测.

\section{5 探针 PIOCI 对 $\mathrm{HOCl}$ 的选择性研究}

面对多样的检测环境, 较好的选择性是探针进行有 效检测目标分子的首要条件. 因此选用探针 PIOCI (10 $\mu \mathrm{mol} / \mathrm{L})$ 和不同的生物相关分析物, 在 PBS 缓冲溶液 (20 $\mathrm{mmol} / \mathrm{L}, \mathrm{pH}=7.4,30 \% \mathrm{EtOH})$ 中进行苂光发射光谱测试, 并记录 $514 \mathrm{~nm}$ 处的苂光强度变化. 这些分析物包含以 下分子或离子: $\mathrm{Mg}^{2+}, \mathrm{Zn}^{2+}, \mathrm{Ca}^{2+}, \mathrm{Cu}^{2+}, \mathrm{Fe}^{3+}, \mathrm{F}^{-}, \mathrm{NO}_{3}^{-}$, $\mathrm{NO}_{2}^{-}, \mathrm{HS}^{-}, \mathrm{HSO}^{-}$, Cys, $\mathrm{GSH}, \mathrm{H}_{2} \mathrm{O}_{2}, \cdot \mathrm{OH}, \mathrm{O}_{2}^{-\cdot},{ }^{1} \mathrm{O}_{2}$, $t-\mathrm{BuOO}^{-}, \mathrm{NO} \cdot, \mathrm{ONOO}^{-}, \mathrm{HOBr}$ 和 $\mathrm{HOCl}$. 分析物加入量 均为 1 倍量. 如图 3 所示, 除了加入 $\mathrm{HOCl}$ 外, 其它分析 

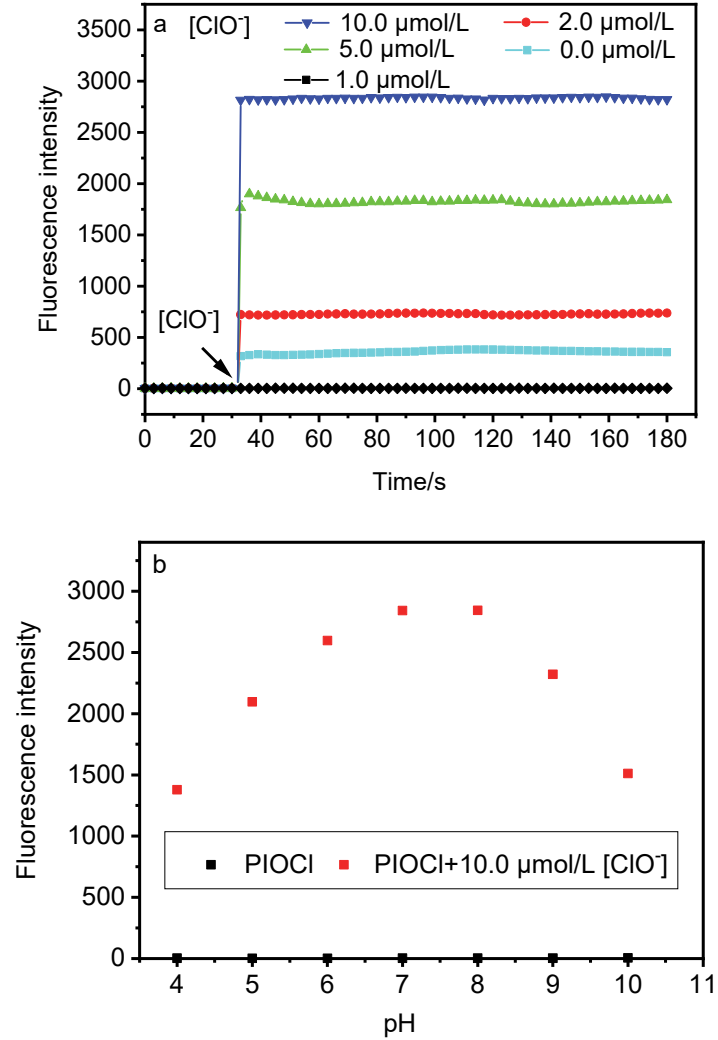

图 2 (a) 苂光强度随时间变化曲线 $\left(\lambda_{\mathrm{ex}}=420 \mathrm{~nm}\right.$, 探针 PIOCI $10 \mu \mathrm{mol} / \mathrm{L}, \mathrm{HOCl}$ 分别为 $0,1.0,2.0,5.0$ 和 $10 \mu \mathrm{mol} / \mathrm{L}$ ), 以及(b) 探针 PIOCl $(10 \mu \mathrm{mol} / \mathrm{L})$ 及探针 PIOCl $(10 \mu \mathrm{mol} / \mathrm{L})$ 与 $\mathrm{HOCl}(10$ $\mu \mathrm{mol} / \mathrm{L}$ )苂光响应受 $\mathrm{pH}$ 的影响

Figure 2 (a) Time-dependent fluorescent intensities of probe PIOCl treated with $\mathrm{HOCl}\left(\lambda_{\mathrm{ex}}=420 \mathrm{~nm}\right.$, PIOCl $10 \mu \mathrm{mol} / \mathrm{L}$, $\mathrm{HOCl} 0,1.0,2.0,5.0$, and $10 \mu \mathrm{mol} / \mathrm{L}$ ), and (b) plot of fluorescence intensity of probe PIOCI $(10.0 \mu \mathrm{mol} / \mathrm{L})$ in the absence/ presence of $\mathrm{HOCl}(10 \mu \mathrm{mol} / \mathrm{L})$ at different $\mathrm{pH}$

物对探针 PIOCI 的苂光强度几乎没有变化. 实验结果说 明荧光探针 PIOCI 对 $\mathrm{HOCl}$ 具有较高的选择性.

\section{6 探针 $\mathrm{PIOCI}$ 对 $\mathrm{HOCl}$ 的反应机理}

为了探究探针 PIOCI 与 $\mathrm{HOCl}$ 的响应机制, 对探针 PIOCI 与 $\mathrm{HOCl}$ 反应后产物的高分辨质谱、光谱性质、 核磁共振波谱和理论计算进行了研究。根据文献 [24-25], 探针分子中含有的吩噻嗪结构是 $\mathrm{HOCl}$ 的良好 识别基团，其反应机制是硫醚结构被氧化为亚砜 $[\mathbf{P I O C I}+\mathrm{O}]$ 或砜 $[\mathbf{P I O C I}+2 \mathrm{O}]$ 结构, 然而在高分辨质谱 中没有找到亚砜 $[\mathbf{P I O C I}+\mathrm{O}] \mathrm{m} / \mathrm{z} 401.1086$ 或砜[PIOCI $+2 \mathrm{O}] \mathrm{m} / \mathrm{z} 417.1035$ 的相关质谱峰. 对文献报道的以吩 噻嗪为母体的次氯酸荧光探针进行分析, 发现吩噻嗪衍 生物与次氯酸的反应性与共轭基团有很大关系. 当吩噻 嗪结构与吸电子基团共轭时, 会降低吩噻溙结构的电子 分布 ${ }^{[25,34]}$, 从而降低吩噻嗪中硫醚结构与次氯酸的反应 活性，并且当新引入的官能团具有更高的反应活性时，
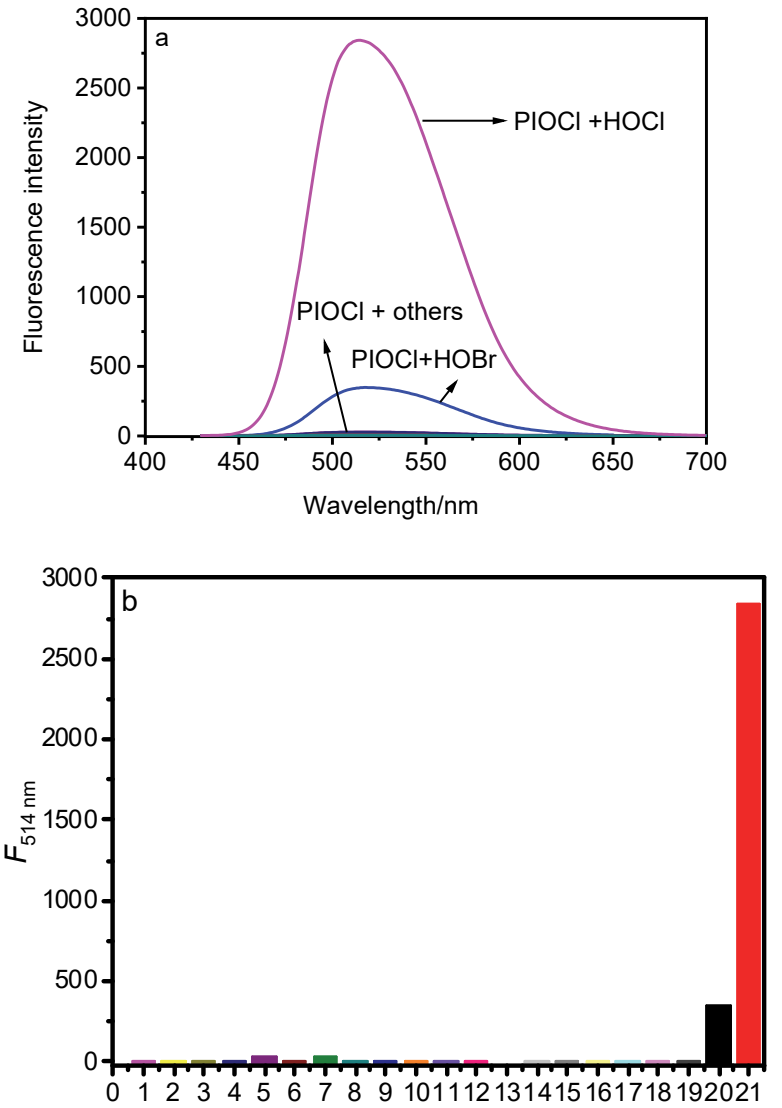

图 3 (a)探针 PIOCI 与各种分析物相互作用后的荧光光谱图, 以及(b)在 $514 \mathrm{~nm}$ 处各种分析物的荧光响应强度

Figure 3 (a) Fluorescence spectra of probe PIOCI in the presence of different biological relevant analytes and (b) fluorescent intensity changes of probe PIOCl in the presence of different biological relevant analytes at $514 \mathrm{~nm}$

The numbers represent: (1) probe PIOCI $\left(1.0 \times 10^{-5} \mathrm{~mol} / \mathrm{L}\right),(2) \mathrm{MgCl}_{2}$, (3) $\mathrm{Zn}\left(\mathrm{NO}_{3}\right)_{2}$, (4) $\mathrm{Ca}\left(\mathrm{NO}_{3}\right)_{2}$, (5) $\mathrm{Cu}\left(\mathrm{NO}_{3}\right)_{2}$, (6) $\mathrm{Fe}\left(\mathrm{NO}_{3}\right)_{3}$, (7) $\mathrm{NaF}$, (8) $\mathrm{NaNO}_{3}$, (9) $\mathrm{NaNO}_{2}$, (10) $\mathrm{Na}_{2} \mathrm{~S}$, (11) Cys, (12) GSH, (13) $\mathrm{H}_{2} \mathrm{O}_{2}$, (14) $\bullet \mathrm{OH}$, (15) $\bullet \mathrm{O}_{2}^{-},(16){ }^{1} \mathrm{O}_{2},(17) t-\mathrm{BuOO}^{-}$, (18) $\mathrm{NO} \cdot$, (19) $\mathrm{ONOO}^{-}$, (20) $\mathrm{HOBr}$ (21) $\mathrm{NaClO} . \lambda_{\text {ex }}=420 \mathrm{~nm}$

次氯酸会与新的位点进行反应 ${ }^{[35-36]}$. 通过对高分辨质谱 分析，发现 $[\mathbf{P I O C I}+\mathrm{Cl}] \mathrm{m} / \mathrm{z} 419.0747$ 对应的 419.07374 质谱峰, 如图 4 所示. 实验结果表明, 探针 PIOCI 与 $\mathrm{HOCl}$ 的响应机制是发生氯代反应. 目前文献报道基于 $\mathrm{HOCl}$ 的氯代反应主要有两种: (1)在酸性条件下, $\mathrm{Cl}^{+}$与 探针分子的芳香体系发生亲电取代反应 ${ }^{[26] ; ~(2) ~} \mathrm{Cl}^{+}$与探 针分子中的羟基反应形成 $\mathrm{O}-\mathrm{Cl}$ 键. 这种 $\mathrm{O}-\mathrm{Cl}$ 键并不 稳定, 在酸性条件下转换为原探针分子[27-28]. 因此应用 紫外可见光谱研究了探针 $\mathbf{P I O C l}$ 与 $\mathrm{HOCl}$ 反应后加入酸 的变化情况. 如图 5 所示, 加入酸后, 其最大吸收峰并 未发生太大变化, 表明探针 PIOCl 与 $\mathrm{HOCl}$ 的反应并未 形成 $\mathrm{O}-\mathrm{Cl}$ 键, 而是由 $\mathrm{Cl}^{+}$引发的亲电取代反应. 根据 以上实验结果提出了探针 $\mathbf{P I O C l}$ 与 $\mathrm{HOCl}$ 的响应机制 (Scheme 1). 此外, 核磁共振光谱结果如图 6 所示, 探针 PIOCl 与 $\mathrm{HOCl}$ 反应后, ${ }^{1} \mathrm{H}$ NMR 谱中 $\mathrm{H}_{\mathrm{c}}(\delta$ 7.042) 的质 
子峰消失, 䒢酮环上 $\mathrm{H}_{\mathrm{d}}(\delta 6.911)$ 和 $\mathrm{H}_{\mathrm{e}}(\delta 7.569)$ 的质子信 号峰同时向低场 $\delta 7.162$ 和 7.609 移动, 表明探针 PIOCI 的氯代反应发生在 $\mathrm{Hc}$ 质子上.

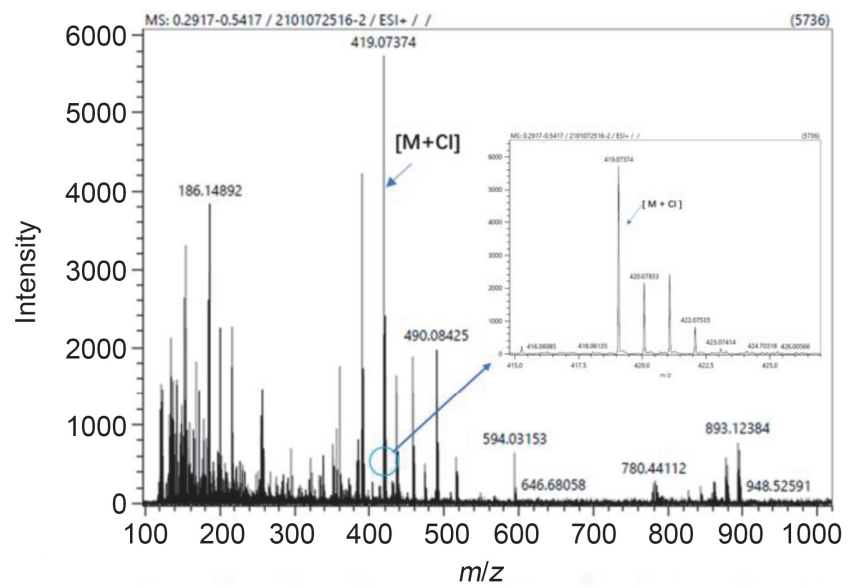

图 4 探针 PIOCl 与 $\mathrm{HOCl}$ 作用后的高分辨质谱

Figure 4 HRMS spectrum of PIOCl with $\mathrm{HOCl}$

为了进一步研究探针 PIOCI 对 $\mathrm{HOCl}$ 的识别机制, 使用 Gaussian 09 程序, 采用 DFT/B3LYP/6-31G(d,p)基 组对探针 PIOCI 和探针与 $\mathrm{HOCl}$ 反应后的产物 PIOCC 进行结构优化, 分别得到相应的轨道电荷分布图, 如图 7 所示. 计算出探针 PIOCI 分子 HOMO 轨道与 LUMO 轨道的能级差对应的吸收波长为 $426 \mathrm{~nm}$ (实验值为 435 $\mathrm{nm}$ ), 反应后产物 PIOCC 的 HOMO 与 LUMO 轨道的能 级差对应的吸收波长为 $404 \mathrm{~nm}$ (实验值为 $400 \mathrm{~nm}$ ). 实验 结果与理论计算相一致, 进一步证实了探针 PIOCI 与 $\mathrm{HOCl}$ 的响应机制.

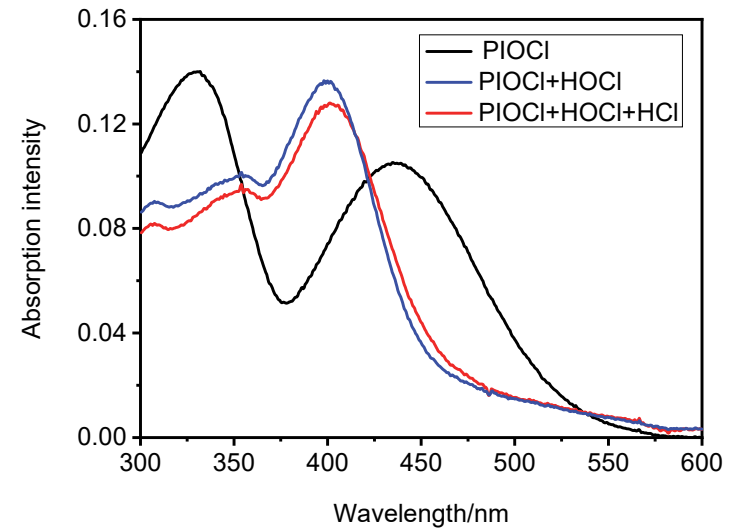

图 5 探针 PIOCI 与 $\mathrm{HOCl}$ 作用后受酸影响的紫外可见吸收光 谱图

Figure 5 UV-Vis absorption spectra of probe PIOCl (10.0 $\mu \mathrm{mol} / \mathrm{L})$ to $\mathrm{HOCl}(10.0 \mu \mathrm{mol} / \mathrm{L})$ in the absence and presence of $\mathrm{H}^{+}$

\section{7 探针 PIOCI 在 HeLa 细胞中的荧光成像}

荧光光谱测试表明, 探针 PIOCI 对 $\mathrm{HOCl}$ 具有较高 的灵敏度和选择性，能在生理条件下实现对 $\mathrm{HOCl}$ 的快 速响应. 为此, 进一步开展了 $\mathrm{HOCl}$ 生物成像研究. 在 苂光成像实验前, 用噻唑蓝(MTT)法测试了 PIOCI 在人 宫颈癌 HeLa 活细胞中的毒性. 结果表明, 探针在 $0 \sim 20$ $\mu \mathrm{mol} / \mathrm{L}$ 范围内对人宫颈癌 HeLa 细胞没有表现出显著的 细胞毒性. 随后采用苂光显微镜探究了探针 PIOCI 在 $\mathrm{HeLa}$ 细胞中对内源和外源 $\mathrm{HOCl}$ 的苂光成像. 将 10 $\mu \mathrm{mol} / \mathrm{L}$ 探针 PIOCI 溶液加入到预先培养好的 $\mathrm{HeLa}$ 细胞 中, 在培养基中于 $37{ }^{\circ} \mathrm{C}$ 培养 $30 \mathrm{~min}$, 用 $\mathrm{PBS}$ 缓冲液洗 涤后进行细胞苂光成像, 结果发现在 HeLa 活细胞中表
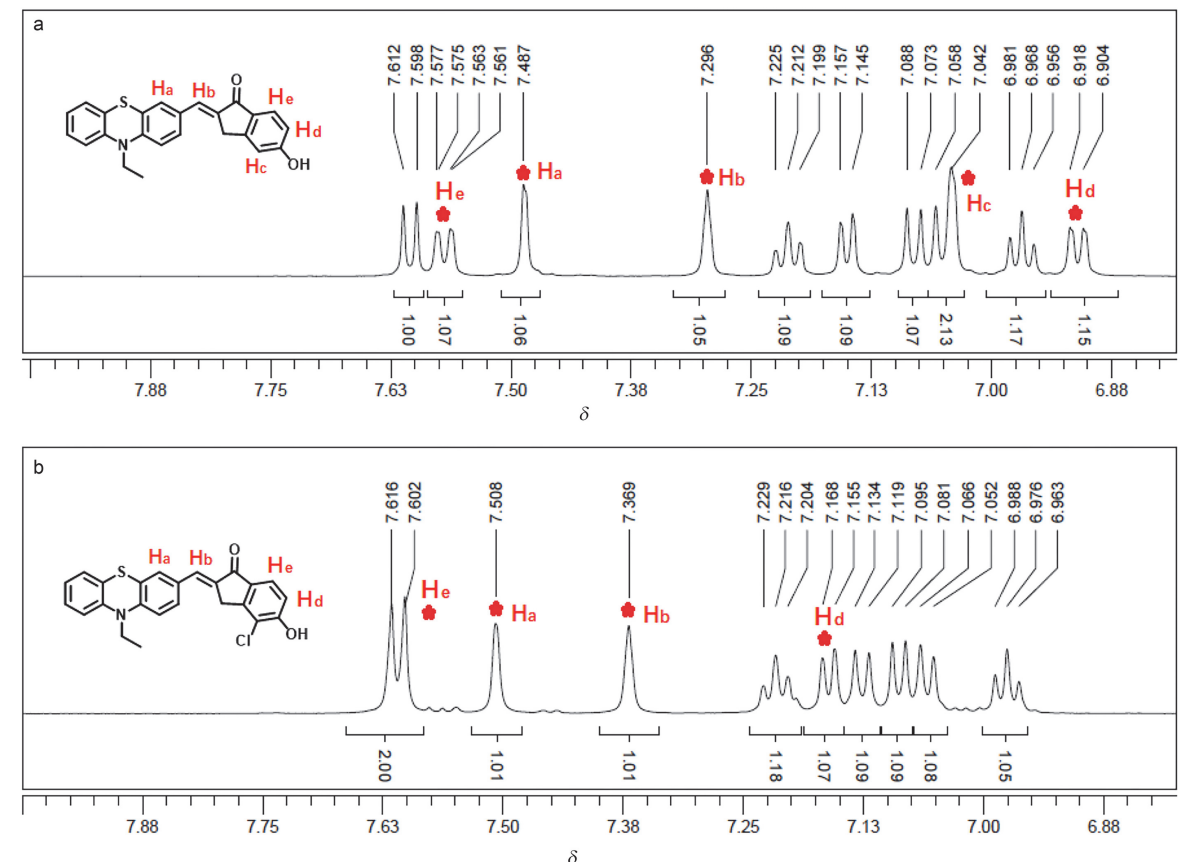

图 6 探针 $\mathrm{PIOCl}$ 与 $\mathrm{HOCl}$ 反应后 ${ }^{1} \mathrm{H}$ NMR 谱

Figure $6{ }^{1} \mathrm{H}$ NMR spectrum of PIOCl with $\mathrm{HOCl}$ 

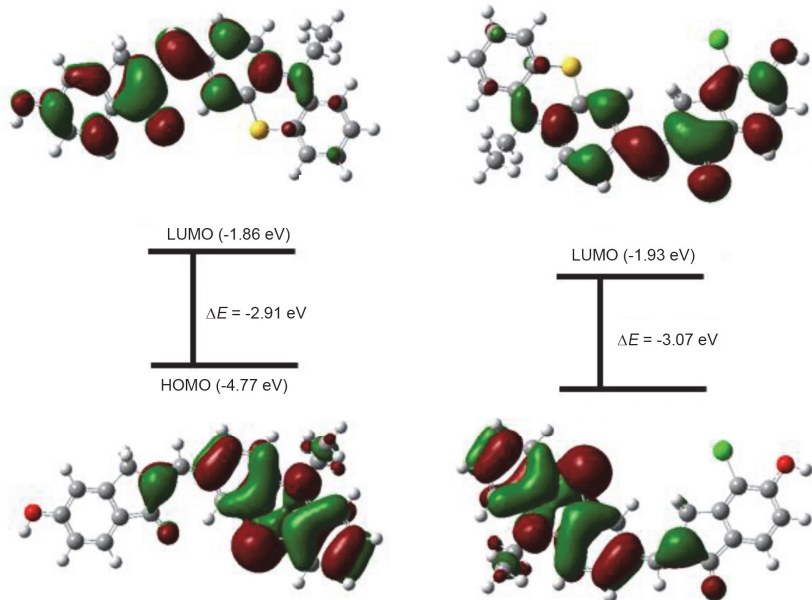

$\mathrm{PIOCI}$

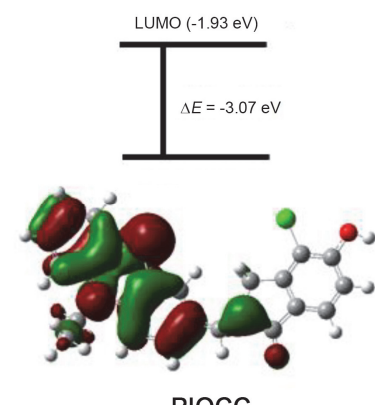

PIOCC

图 7 探针 PIOCI 和 PIOCC 的优化分子结构和前线轨道能量

Figure 7 The Optimized structures and frontier orbital energy of PIOCI and PIOCC

现出微弱的荧光信号, 如图 $8 \mathrm{a} \sim 8 \mathrm{~b}$ 所示. 这是因为人宫 颈癌 HeLa 细胞处于高水平的氧化应激状态, 导致活性 氧(如 $\mathrm{HOCl}, \mathrm{ONOO}^{-}, \mathrm{H}_{2} \mathrm{O}_{2}$ )在癌细胞中过渡表达 ${ }^{[29-30]}$. 随后, 再向上述含 PIOCl 的细胞培养液中加入 $\mathrm{NaClO}$ $(10 \mu \mathrm{mol} / \mathrm{L})$, 并在 $37^{\circ} \mathrm{C}$ 下孵育 $30 \mathrm{~min}$, 发现 HeLa 活细 胞呈现出明亮的黄色荧光, 如图 $8 \mathrm{c} \sim 8 \mathrm{~d}$ 所示, 证实了探 针 PIOCI 能够对活细胞中内源和外源性 $\mathrm{HOCl}$ 水平进行 生物影像, 表明探针 PIOCI在生物学上具有潜在的应用 价值.
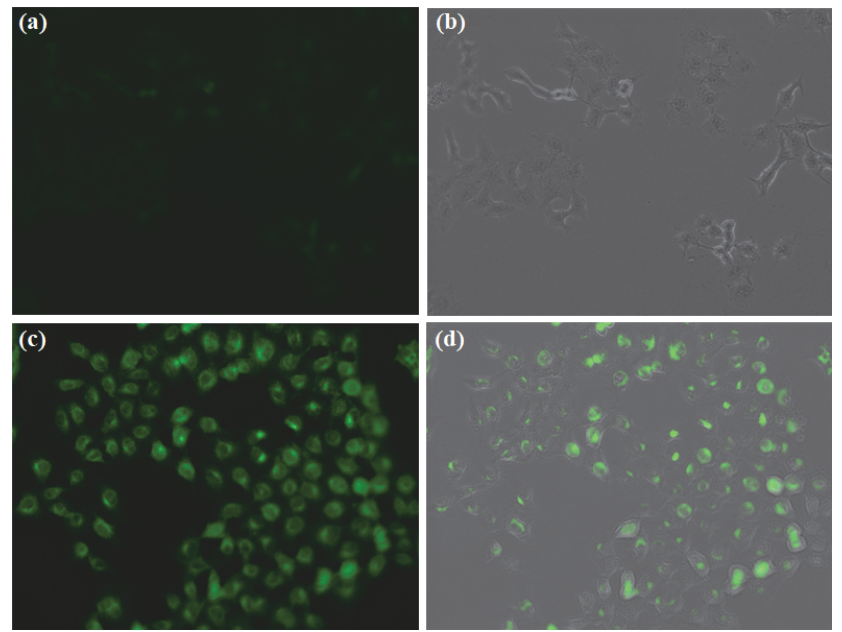

图 8 探针 PIOCI 在 HeLa 活细胞中的荧光成像

Figure 8 Live cell fluorescence imaging of probe PIOCl in living HeLa cells

$(\mathrm{a} \sim \mathrm{b})$ Living HeLa cells incubation with PIOCI $(10 \mu \mathrm{mol} / \mathrm{L})$ for 30 $\mathrm{min}$, and $(\mathrm{c} \sim \mathrm{d})$ living HeLa cells incubation with PIOCl $(10 \mu \mathrm{mol} / \mathrm{L})$ and $\mathrm{HOCl}(10 \mu \mathrm{mol} / \mathrm{L})$ for $30 \mathrm{~min}$

\section{2 结论}

通过在狮酮分子引入具有强给电子吩噻嗪基团, 设
计合成出一种新型超灵敏次氯酸苂光探针. 该探针在生 理条件下对 $\mathrm{HOCl}$ 具有良好的选择性和灵敏性，测限达 到 $1.40 \mathrm{nmol} / \mathrm{L}$, 能够对 $0.25 \mu \mathrm{mol} / \mathrm{L}$ 的 $\mathrm{HOCl}$ 裸眼观察, 响应时间小于 $3 \mathrm{~s}$. 细胞成像实验表明, 该探针可用于细 胞内源性和外源性 $\mathrm{HOCl}$ 的检测. 光谱实验和理论计算 结果表明，探针与次氯酸的响应机制为氯正离子引发的 亲电取代反应机制. 这一成果将为构建新型次氯酸荧光 探针提供新思路.

\section{3 实验部分}

\section{1 仪器与试剂}

所用的化学试剂原料均为市售化学纯, 所用溶剂为 分析纯或光谱纯. 基本必需培养基 Dulbecco's Modified Eagle's Medium (DMEM, High glucose)和胎牛血清(FBS) 购自 Invitrogen(沃尔瑟姆, 马萨诸塞州, 美国). 使用 SPECORD S600 spectrometer 紫外分光光度计(耶拿, 德 国)记录吸收光谱. 在 F7000 苂光光谱仪(日立, 日本)上 收集荧光光谱. 采用 Aglient 7250 (Agilent, 美国)\& JEOL-JMS-T100LP AccuTOF(日本电子株式会社, 日本 东京) 液质联用仪进行高分辨质谱(HRMS) 分析. ${ }^{1} \mathrm{H}$

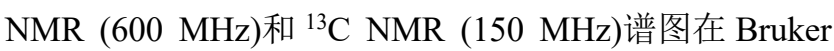
AVANCE-600M spectrometer (布鲁克, 瑞士)上进行, 以 四甲基硅烷为内标. 细胞苂光图像在 $\mathrm{EVOS} \otimes \mathrm{FL}$ Auto Imaging System (Thermo Fisher Scientific, 沃尔瑟姆, 马 萨诸塞州, 美国)上获得.

\section{2 探针(Z)-2-( $N$-乙基-3-亚甲基吩噻嗪基)-5-羟基-1- 狮酮(PIOCI)的合成}

在 $100 \mathrm{~mL}$ 圆底烧瓶中, 氮气保护下加入 $3.0 \mathrm{~mL}$ $\mathrm{DMF}$, 冰盐浴冷却到 $0{ }^{\circ} \mathrm{C}$, 缓慢滴加 $2.0 \mathrm{~mL} \mathrm{POCl}_{3}$, 在 此温度下搅拌 $30 \mathrm{~min}$. 将 $N$-乙基吩噻嗪(1.6 g, $7.0 \mathrm{mmol}$ ) 溶解到 $5.0 \mathrm{~mL} \mathrm{~N}, N$-二甲基甲酰胺(DMF)中, 然后缓慢加 入到反应瓶. 揽拌 $10 \mathrm{~min}$ 后，加热至 $60{ }^{\circ} \mathrm{C}$ ，反应 $12 \mathrm{~h}$. 将反应液缓慢倒入盛有 $100 \mathrm{~mL}$ 冰水的烧杯里, 不断摚 拌, 用饱和 $\mathrm{Na}_{2} \mathrm{CO}_{3}$ 溶液调节 $\mathrm{pH}$ 至中性, 二氯甲烷萃取, 有机相用无水硫酸钠干燥后旋蒸, 用乙酸乙酯/石油醚 $(V: V=1 ： 10)$ 柱层析分离, 得到深黄色固体 $N$-乙基-3甲酰基- $10 H$-吩噻嗪(M1) $1.00 \mathrm{~g}$, 产率 56\%. m.p. 97.0 $97.8{ }^{\circ} \mathrm{C}$ (Lit. ${ }^{[31]}$ m.p. $\left.98{ }^{\circ} \mathrm{C}\right) ;{ }^{1} \mathrm{H}$ NMR $\left(\mathrm{CDCl}_{3}, 600 \mathrm{MHz}\right)$ $\delta: 9.78(\mathrm{~s}, 1 \mathrm{H}), 7.63 \sim 7.61(\mathrm{~m}, 1 \mathrm{H}), 7.56 \sim 7.561(\mathrm{~m}, 1 \mathrm{H})$, $7.16 \sim 7.14(\mathrm{~m}, 1 \mathrm{H}), 7.10 \sim 7.08(\mathrm{~m}, 1 \mathrm{H}), 6.96 \sim 6.94(\mathrm{~m}$, $1 \mathrm{H}), 6.90 \sim 6.88(\mathrm{~m}, 2 \mathrm{H}), 3.98 \sim 3.96(\mathrm{~m}, 2 \mathrm{H}), 1.44(\mathrm{t}, J=$ $6.6 \mathrm{~Hz}, 3 \mathrm{H})$.

在 $100 \mathrm{~mL}$ 圆底烧瓶中, 氮气保护下将 5-羟基-1-茚 酮(1.0 mmol, $0.148 \mathrm{~g}$ )、3-甲酰基- $N$-乙基吩噻嗪( $0.255 \mathrm{~g}$, $1.0 \mathrm{mmol})$ 和 $\mathrm{NaOH}(2.0 \mathrm{mmol}, 0.08 \mathrm{~g})$ 加入到 $20.0 \mathrm{~mL}$ 干 
燥乙醇中, 在此温度下搅拌 $30 \mathrm{~min}$. 随后将温度逐渐升 至 $90{ }^{\circ} \mathrm{C}$ 反应 $10.0 \mathrm{~h}$, 反应完全后产生大量黄色沉淀. 冷 却至室温, 用冰乙酸调节 $\mathrm{pH}$ 中性, 过滤, 用冰乙醇重复 洗涤, 干燥后得黄色固体化合物 PIOCI, 产率 $85 \%$. m.p. $278.5 \sim 280.0{ }^{\circ} \mathrm{C} ;{ }^{1} \mathrm{H}$ NMR $\left(600 \mathrm{MHz}, \mathrm{DMSO}-d_{6}\right) \delta$ : $1.32(\mathrm{t}, J=7.2 \mathrm{~Hz}, 3 \mathrm{H}), 3.95 \sim 3.97(\mathrm{~m}, 4 \mathrm{H}), 6.91(\mathrm{~d}, J=$ $8.4 \mathrm{~Hz}, 1 \mathrm{H}), 6.97$ (t, $J=7.8 \mathrm{~Hz}, 1 \mathrm{H}), 7.04 \sim 7.06(\mathrm{~m}, 2 \mathrm{H})$, 7.08 (d, $J=9.0 \mathrm{~Hz}, 1 \mathrm{H}), 7.15(\mathrm{~d}, J=7.2 \mathrm{~Hz}, 1 \mathrm{H}), 7.21(\mathrm{t}$, $J=7.8 \mathrm{~Hz}, 1 \mathrm{H}), 7.30$ (s, 1H), 7.49 (s, 1H), 7.57 (dd, $J=7.2$ $\mathrm{Hz}, 1.2 \mathrm{~Hz}, 1 \mathrm{H}), 7.61(\mathrm{~d}, J=8.4 \mathrm{~Hz}, 1 \mathrm{H}) ;{ }^{13} \mathrm{C}$ NMR $(100$ MHz, DMSO-d $) \delta: 12.99,32.22,41.82,112.48,115.77$, $116.13,116.65,122.51,123.38,123.43,126.01,127.56$, $128.31,128.92,129.73,129.92,130.41,131.21,134.54$, 145.60, 153.24, 164.59, 191.76. HRMS (EI) calcd for $\mathrm{C}_{24} \mathrm{H}_{19} \mathrm{NO}_{2} \mathrm{~S} 385.11310$, found 385.11308.

\section{3 探针 $\mathrm{PIOCI}$ 的光谱性质和荧光响应研究}

将 PIOCI 溶解在 DMF 中得到 $100 \mathrm{mmol} / \mathrm{L}$ 储备溶 液, 避光保存. 以次氯酸钠的量来提供 $\mathrm{HOCl}$ 来源. 将 相应待分析物溶解于蒸馏水中, 以及按照文献获得次溴 酸 ${ }^{[32]}$ 、活性氧和氮客体的配制 ${ }^{[33]}$. 按标准方法制备磷酸 盐缓冲液( $\mathrm{PBS}, 20 \mathrm{mmol} / \mathrm{L}, \mathrm{pH}=7.4$ ). 使用 $\mathrm{PBS}$ 缓冲溶 液 $(20 \mathrm{mmol} / \mathrm{L}, \mathrm{pH}=7.4,30 \% \mathrm{EtOH})$ 将新鲜储备溶液稀释 至所需浓度. 没有特别说明情况下, 苂光光谱测量的激 发波长为 $420 \mathrm{~nm}$, 激发狭缝宽度均为 $5 \mathrm{~nm}$, 发射狭缝宽 度均为 $2.5 \mathrm{~nm}$, PMT Voltage $600 \mathrm{~V}$.

\section{4 检测限}

对探针 PIOCI (10 $\mu \mathrm{mol} / \mathrm{L})$ (空白组)在 $420 \mathrm{~nm}$ 激发 下的荧光强度进行 10 次测定, 获得空白组在 $514 \mathrm{~nm}$ 处 荧光强度的标准偏差. 根据苂光响应中 PIOCI (10 $\mu \mathrm{mol} / \mathrm{L})$ 与 $\mathrm{HOCl}(0 \sim 6.0 \mu \mathrm{mol} / \mathrm{L})$ 的线性相关计算检测限 $(\mathrm{DL})^{[10]}$. 计算公式如下:

\section{$\mathrm{DL}=3 \sigma / k$}

其中 $\sigma$ 是空白测量的标准偏差, $k$ 是探针 PIOCI (10 $\mu \mathrm{mol} / \mathrm{L})$ 与 $\mathrm{HOCl}(0 \sim 6.0 \mu \mathrm{mol} / \mathrm{L})$ 反应后 $514 \mathrm{~nm}$ 处苂光 强度与不同 $\mathrm{HOCl}$ 浓度之间的斜率.

\section{5 探针 PIOCI 对 $\mathrm{HOCl}$ 的响应机制}

探针 PIOCl $(100 \mu \mathrm{mol} / \mathrm{L})$ 与 $\mathrm{HOCl}(100 \mu \mathrm{mol} / \mathrm{L})$ 在 $\mathrm{PBS}$ 缓冲溶液 $(20 \mathrm{mmol} / \mathrm{L}, \mathrm{pH}=7.4,30 \% \mathrm{EtOH})$ 中 $25{ }^{\circ} \mathrm{C}$ 反应 $30 \mathrm{~min}$, 然后加入少量乙酸乙酯 $(15 \mathrm{~mL} \times 3)$ 萃取, 收集有机相, 旋转蒸发除去有机溶剂后, 对产物进行高 分辨质谱和核磁共振光谱 ${ }^{1} \mathrm{H}$ NMR 表征.

\section{6 细胞培养和 MTT 分析}

HeLa 细胞(人宫颈癌细胞)购自 KeyGen Biotech(南 京, 中国). 细胞培养在添加了体积分数为 $10 \%$ 胎牛血
清、 $80 \mathrm{U} / \mathrm{mL}$ 青霉素和 $0.08 \mathrm{mg} / \mathrm{mL}$ 链霉素的 MEM 中, 在体积分数为 $5 \% \mathrm{CO}_{2}$ 培养箱中 $37{ }^{\circ} \mathrm{C}$ 培养 $24 \mathrm{~h}$.

采用噻唑蓝(MTT)法测定探针 PIOCI对以上三种活 细胞的细胞毒性. 噻唑蓝购买自 Solarbio (索莱宝, 北 京, 中国). HeLa 细胞接种在 96 孔板中, 每孔密度 $1 \times 10^{4}$, 在 $5 \% \mathrm{CO}_{2}$ 培养箱中 $37{ }^{\circ} \mathrm{C}$ 培养过夜. 将不同浓 度的 PIOCI $(0,5.0,10.0,15.0,20.0 \mu \mathrm{mol} / \mathrm{L})$ 加入到细胞 中继续培养 $24 \mathrm{~h}$, 然后将噻唑蓝 $(20 \mu \mathrm{mol} / \mathrm{L}, 5 \mathrm{mg} / \mathrm{mL}$ )加 入培养基继续孵育 $4 \mathrm{~h}$. 小心地去除培养基，每孔加入 $150 \mu \mathrm{L}$ 二甲基亚砜并摇 $10 \mathrm{~min}$ 以完全溶解，最后用多 功能酶标仪(Thermo Fisher, MA,USA)测定 $490 \mathrm{~nm}$ 处的 吸光值, 计算细胞存活率.

\section{7 成像实验}

苂光成像实验前一天, 在共聚焦培养血中培养 HeLa 细胞, 共设置三组. 对照组只用 PBS 处理细胞. 实 验组 1 与 PIOCI $(10 \mu \mathrm{mol} / \mathrm{L})$ 共培养 $0.5 \mathrm{~h}$. 实验组 2 与 PIOCl $(10 \mu \mathrm{mol} / \mathrm{L})$ 预处理 $0.5 \mathrm{~h}$, 再加入 $\mathrm{HOCl}(10$ $\mu \mathrm{mol} / \mathrm{L})$ 共培养 $0.5 \mathrm{~h}$. 采用荧光显微镜进行细胞成像观 察.

\section{8 理论计算}

采用密度泛函理论(DFT), 在 Gaussian09 程序中用 DFT/B3LYP/6-31G(d,p) 基组对 PIOCI 和 PIOCC 分别进 行了结构优化, 得到了最高占据分子轨道(HOMO)和最 低未占据分子轨道(LUMO)的能级.

辅助材料(Supporting Information) 化合物 M1 和探针 PIOCI 的相关表征谱图, 实验方法以及部分荧光谱图和 细胞实验数据. 这些材料可以免费从本刊网站(http:// sioc-journal. cn/)上下载.

\section{References}

[1] Zielonka, J.; Joseph, J.; Sikora, A.; Hardy, M.; Ouari, O.; Vasquez-Vivar, J.; Cheng, G.; Lopez, M.; Kalyanaraman, B. Chem. Rev. 2017, 117, 10043.

[2] Wu, D.; Chen, L. Y.; Xu, Q. L.; Chen, X. Q.; Yoon, J. Y. Acc. Chem. Res. 2019, 52, 2158.

[3] Wang Y. B.; Zhao B. X. Chin. J. Org. Chem. 2016, 36, 1539 (in Chinese).

(王延宝, 赵宝祥, 有机化学, 2016, 36, 1539.)

[4] Yuan, L.; Wang, L.; Agrawalla, B. K.; Park, S. J.; Zhu, H.; Sivaraman, B.; Peng, J.; Xu, Q. H.; Chang, Y. T. J. Am. Chem. Soc. 2015, 137,5930

[5] Pattison, D. I.; Davies, M. J. Chem. Res. Toxicol. 2001, 14, 1453.

[6] Wei, P.; Liu, L.; Yuan, W.; Yang, J.; Li, R.; Yi, T. Sci. China Chem. 2020, 63, 1153.

[7] Nathan, C.; Cunningham-Bussel, A. Nat. Rev. Immunol. 2013, 13, 349.

[8] Ma, C.; Zhong, G.; Zhao, Y.; Zhang, P.; Fu, Y.; Shen, B. Spectrochim. Acta A 2020, 240, 118545 .

[9] Yue, Y.; Huo, F.; Yin, C.; Escobedo, J. O.; Strongin, R. M. Analyst 2016, 141,1859 . 
[10] Zhang, Y. R.; Liu, Y.; Feng, X.; Zhao, B. X. Sens. Actuators B: Chem. 2017, 240, 18.

[11] Yu Q., Chen X. L., Liu H., Zhang Q. L. Chin. J. Org. Chem. 2020, 40, 1206 (in Chinese) (余青，陈晓丽，刘华，张奇龙，有机化学, 2020, 40, 1206.)

[12] Zhu, H.; Fan, J.; Wang, J.; Mu, H.; Peng, X. J. Am. Chem. Soc. 2014, 136, 12820,

[13] He, L.; Xiong, H.; Wang, B.; Zhang, Y.; Wang, J.; Zhang, H.; Li, H.; Yang, Z.; Song, X. Anal. Chem. 2020, 92, 11029,

[14] Radi, R.; Beckman, J. S.; Bush, K. M.; Freeman, B. A. J. Biol. Chem. 1991, 266, 4244,

[15] Koide, Y.; Urano, Y.; Kenmoku, S.; Kojima, H.; Nagano, T. J. Am. Chem. Soc. 2007, 129, 10324,

[16] Lou, Z.; Li, P.; Han, K. Acc. Chem. Res. 2015, 48, 1358,

[17] Zhang, H.; Liu, J.; Liu, C.; Yu, P.; Sun, M.; Yan, X.; Guo, J.-P.; Guo, W. Biomaterials 2017, 133, 60,

[18] Jiao C. P., Liu Y. Y., Lu W. J., Zhang P. P., Wang Y. F. Chin. J. Org. Chem. 2019, 39, 591 (in Chinese).

(矫春鹏, 刘媛媛, 路文娟, 张平平, 王延风, 有机化学, 2019, 39, 591.)

[19] Xie, X.; Wu, T.; Wang, X.; Li, Y.; Wang, K.; Zhao, Z.; Jiao, X.; Tang, B. Chem. Commun. 2018, 54, 11965.

[20] Chen, X.; Wang, F.; Hyun, J. Y.; Wei, T.; Qiang, J.; Ren, X.; Shin, I.; Yoon, J. Chem. Soc. Rev. 2016, 45, 2976.

[21] de la Mare, P. B. D.; Hilton, I. C.; Vernon, C. A. J. Chem. Soc. (Resumed) 1960, 4039.

[22] Ren, T.-B.; Xu, W.; Zhang, Q.-L.; Zhang, X.-X.; Wen, S.-Y.; Yi, H.-B.; Yuan, L.; Zhang, X.-B. Angew. Chem. Int. Ed. 2018, 57, 7473.
[23] Wu, M. Y.; Li, K.; Hou, J. T.; Huang, Z.; Yu, X. Q. Org. Biomol. Chem. 2012, 10, 8342.

[24] Weng, J.; Mei, Q.; Zhang, B.; Jiang, Y.; Tong, B.; Fan, Q.; Ling, Q.; Huang, W. Analyst 2013, 138, 6607.

[25] Xiao, H.; Xin, K.; Dou, H.; Yin, G.; Quan, Y.; Wang, R. Chem. Commun. 2015, 51, 1442.

[26] Wang, Y.; Wu, L.; Liu, C.; Guo, B.; Zhu, B.; Wang, Z.; Duan, Q.; Ma, Z.; Zhang, X. J. Mater. Chem. B 2017, 5, 3377.

[27] Zhi, X.; Qian, Y. Talanta 2021, 222, 121503.

[28] Ma, J.; Yan, C.; Li, Y.; Duo, H.; Li, Q.; Lu, X.; Guo, Y. Chem.-Eur. J. 2019, 25, 7168.

[29] Lu, P.; Zhang, X.; Ren, T.; Yuan, L. Chin. Chem. Lett. 2020, 31, 2980.

[30] Hileman, E. O.; Liu, J.; Albitar, M.; Keating, M. J.; Huang, P. Cancer Chemother. Pharmacol. 2004, 53, 209.

[31] Toşa, M.; Paizs, C.; Majdik, C.; Moldovan, P.; Novák, L.; Kolonits, P.; Szabó, É.; Poppe, L.; Irimie, F.-D. J. Mol. Catal. B: Enzym. 2002, 17, 241.

[32] Yu, F.; Song, P.; Li, P.; Wang, B.; Han, K. Chem. Commun. 2012, 48,7735 .

[33] Jia, P.; Zhuang, Z.; Liu, D.; Chen, Y.; Tian, B.; Liu, C.; Li, Z.; Zhu, H.; Yu, Y.; Zhang, X.; Sheng, W.; Huang, S.; Zhu, B. Sens. Actuators $B$ : Chem. 2020, 305, 127460 .

[34] Liu, F.; Du, J.; Song, D.; Xu, M.; Sun, G. Chem. Commun. 2016, $52,4636$.

[35] Xiao, H. D.; Li, J. H.; Zhao, J.; Yin, G.; Quan, Y. W.; Wang, J.; Wang, R. Y. J. Mater. Chem. B 2015, 3, 1633.

[36] Feng, H.; Wang, Y.; Liu, J.; Zhang, Z.; Yang, X.; Chen, R.; Meng, Q.; Zhang, R. J. Mater. Chem. B 2019, 7, 3909.

(Fan, Y.) 